\title{
Size perception by vision and kinesthesia
}

\author{
TATJANA SEIZOVA-CAJIĆ \\ University of Belgrade, Belgrade, Yugoslavia
}

\begin{abstract}
When two sizes, one perceived by vision and the other by kinesthesia, are apparently equal, the physical relationship between them varies: The sizes may be equal, or the visual size may be larger than the kinesthetic size, or vice versa. In this study, the method of cross-modal matching and the method of magnitude production were used to explore the relationship between apparently equal sizes $(5-40 \mathrm{~cm})$ perceived by vision and by kinesthesia. The sizes were linear or circular, and the mode of standard presentation was visual, kinesthetic, or verbal. The size and the direction of the intermodal mismatch varied with the size of the standard. It was also found that an apparent length of movement varied with the direction of movement. In all conditions, the relationship between apparently equal visual and kinesthetic sizes was well approximated by a power function.
\end{abstract}

Different sense modalities can provide us with information about objects' spatial attributes. For example, the length of a table edge can be perceived both by vision and by kinesthesia combined with touch as one slides one's hand along it. But do we get the same information via different modalities? If one closes one's eyes and slides the hand over the edge, will one's impression of the extent be the same as the one obtained by sight? More generally, the question is whether the relationship between physical sizes is veridically represented in the mind if each of the sizes is perceived via different modality.

This research investigates the relationship between apparently equal sizes perceived by vision and by kinesthesia. Kinesthesia, in the broadest sense, may be defined as the perception of the positions and movements of the limbs and other body parts whether self-generated or externally imposed; sensations from the vestibular and visual systems are excluded (Clark \& Horch, 1986). In this research, kinesthetic percepts arise from the active movements of hand and arm.

It was shown that people in certain situations make systematic errors in intermodal length judgment. Jastrow (1886) applied a cross-modal matching procedure to compare apparently equal sizes perceived by vision, by kinesthesia based on hand or arm movements, and by active touch (finger span). The lengths used were $1-11 \mathrm{~cm}$. The results were the following: Subjects matched shorter visual lengths to longer hand or arm movements and to wider finger span. The results were similar regardless of whether the standard

This article is a version of the author's master's thesis submitted to the University of Belgrade. The author thanks her supervisor, Dejan Todorović, for the education and help he generously provided. The author is also grateful for the excellent working conditions in the Laboratory for Experimental Psychology in Belgrade. The author's thanks also go to Myron Braunstein and two anonymous reviewers for very useful comments on an earlier version of the manuscript, to Branka Spehar for comments on the final version, and to Nick Karadimas for some valuable computer help. Correspondence should be addressed to T. SeizovaCajić, School of Psychology, University of New South Wales, Kensington, NSW 2052, Australia (e-mail: tatjanas@student.unsw.edu.au). was presented to vision and reproduced by the other two modalities, or vice versa. In other words, two physically different extents, one perceived by kinesthesia and the other by vision, may be apparently equal.

The results of later cross-modal studies of visual and kinesthetic length perception are shown in Table $1 .{ }^{1}$ They do not all replicate Jastrow's results. For example, differences obtained by Jones (1973) were very small when compared with Jastrow's, although the range of standard sizes was similar. Laszlo, Begg, and Sainsbury (1994) consistently obtained results opposite to Jastrow's: an overestimation of kinesthetic standard when the match was visual, under a variety of conditions and with complex, circular, and linear stimuli (for references, see Table 1). Intermodal mismatch exists, but it is obviously not stable: It varies in size and in sign, both within and between studies. The disagreement is larger for lengths up to approximately $15 \mathrm{~cm}$ than for longer lengths.

That the results of intermodal comparisons are not always the same should not be surprising, given that intramodal variations of size percepts, visual or kinesthetic size illusions, can result from differences in experimental procedures (procedures used in the reference studies are described in Appendix A). A similar point, although for another subject matter, was clearly made by Stevens (1957): "Of course apparent distance, like apparent size, is specific to the experimental situation and is affected by cues of various sorts. It is not to be expected, therefore, that a single power function will govern all judgments of distance" (p. 168). Similarly, we should not expect to obtain the same differences between vision and kinesthesia in all intermodal comparisons. On the other hand, it is possible that, in some studies, the subjects were not responding to length at all, but to the position (e.g., Brown, Knauft, \& Rosenbaum, 1948).

Apart from variations, there should be a constant feature in the relationship between apparently equal visual and kinesthetic sizes: They should be related by power function. This can be predicted on the basis of the direct measurement studies-studies using methods of ratio es- 
Table 1

\begin{tabular}{|c|c|c|c|}
\hline \multicolumn{4}{|c|}{$\begin{array}{l}\text { Differences Between Apparently Equal Sizes } \\
\text { Perceived by Vision and Kinesthesia }\end{array}$} \\
\hline & $\begin{array}{l}\text { Direction of } \\
\text { the Difference }\end{array}$ & $\begin{array}{l}\text { Size of the Difference } \\
\text { for Different Lengths } \\
\text { (approximate \%) }\end{array}$ & Referent Study \\
\hline \multicolumn{4}{|c|}{ Standard line length $(\mathrm{cm})$} \\
\hline $1-11$ & Vst, $\mathrm{m}<\mathrm{Km}, \mathrm{st}$ & $20-140$ & Jastrow (1886) \\
\hline 2.5 and 30.5 & Vst, $\mathrm{m}>\mathrm{Kst}, \mathrm{m}$ & $40-60$ & Raffel (1936) \\
\hline $0.65-10$ & Vst $<\mathrm{Km}$ & $2-50$ & Brown et al. (1948) \\
\hline $10-40$ & $\mathrm{~V}_{\mathrm{st}}<\mathrm{Km} ; \mathrm{Vst}>\mathrm{Km}^{*}$ & $1-7$ & Brown et al. (1948) \\
\hline 15.2 & $\mathrm{Vst}>\mathrm{Km}$ & n.d. & Millar (1972) \\
\hline $2-10$ & Vst, $\mathrm{m}>\mathrm{Km}, \mathrm{st}^{\dagger}$ & $19-65$ & Jones (1973) \\
\hline $2-10$ & $\mathrm{Vst}<\mathrm{Km}^{\dagger}$ & $9-23$ & Jones (1973) \\
\hline 10 & $\mathrm{Vm}>\mathrm{Kst}^{\dagger}$ & 13 & Laszlo et al. (1994) \\
\hline $2-6$ & $\mathrm{Vm}>\mathrm{Km}$ & $15-20$ & Seizova (1995) \\
\hline $6-30$ & $\mathrm{Vst}>\mathrm{Km}$ & $6-18$ & Seizova (1995) \\
\hline \multicolumn{4}{|c|}{ Standard circle diameter $(\mathrm{cm})$} \\
\hline 15 & $\mathrm{Vm}>\mathrm{Kst}^{\dagger}$ & 37 & Laszlo et al. (1994) \\
\hline 12 & $\mathrm{Vst}>\mathrm{Km}$ & 18 & Zelaznik \& Lantero (1996) \\
\hline
\end{tabular}

Note-V, visual; K, kinesthetic; st, standard; m, match; n.d., no data. *Depending on the direction and orientation of the standard and the match. 'Passive movement.

timation or production, or magnitude estimation or production, or a combination of these (see Stevens, 1957). The logic of the prediction is the following: If the subjective length scale within each of the two modalities is related to the physical scale by particular function (which is power function), then we can calculate the function relating the two modalities - cross-modal matching function. Specifically, it is predicted that the exponent of the cross-modal matching function will be approximately equal to the ratio of the exponents of the two functions (Stevens, 1971).

The exponent for length perceived by vision is close to 1 , according to Stevens and Guirao (1963); Rule (1969) has found an exponent of 1.09. An exponent of 1.05 was obtained for kinesthesia when movement provided the only information about length (Ronco, 1963). In other words, subjective scales for length are almost proportional to the physical scale: When asked to halve the lines they see or feel by movement, subjects are rather accurate in performing the task. Exponents for cross-modal matching function should equal the ratio of the two exponents; therefore, the exponent for the function relating visual and kinesthetic lengths should be close to 1 as well (i.e., these lengths should be almost proportional).

Note that specifying the exponent of the function relating apparently equal visual and kinesthetic sizes tells us nothing about the veridicality. Huge mistakes are still possible: For example, we could feel that 5 and $10 \mathrm{~cm}$ seen are the same as 10 and $20 \mathrm{~cm}$ felt by kinesthesia, respectively. In order to capture the veridicality, we need to know the constant as well as the exponent.

In summary, while attempting to establish the relationship between visual and kinesthetic sizes, we can expect that (1) the difference between apparently equal visual and kinesthetic sizes will vary, and (2) the relationship between apparently equal kinesthetic and visual lengths will be described by power function, with exponent(s) close to 1 .
The motivation for this research was to provide more data concerning the constant errors between apparently equal visual and kinesthetic sizes and data on wider range of lengths within an arm reach than were used before. Conditions were varied within and across experiments, allowing this intermodal relationship to be observed in a variety of situations. In addition to this, direction of movement as a possible source of kinesthetic intramodal variations of the apparent size was explored.

Lengths used were up to $40 \mathrm{~cm}$, which is more than in most of the research cited. Kinesthetic percepts were based on active hand and arm movements. There were three experiments with linear stimuli and one with circular stimuli. Methods were magnitude production (Experiment 1) and cross-modal matching (Experiments $2-4$ ). In the analyses, the differences in physical size between apparently equal sizes were tested and the best-fitting functions describing their relationship were calculated.

\section{EXPERIMENT 1}

In cross-modal comparisons of vision and kinesthesia, the standard may be visual and the matches may be kinesthetic $(\mathrm{Vst}-\mathrm{Km})$, or vice versa $(\mathrm{Kst}-\mathrm{Vm})$. Jastrow (1886) used both designs, and he found symmetry between Vst$\mathrm{Km}$ and $\mathrm{Kst}-\mathrm{Vm}$ situations: Visual lengths were smaller than apparently equal kinesthetic lengths in both designs. Results of later studies were equivocal: Asymmetry appeared in some (e.g., the absolute error was larger in the Vst-Km situation than in the Kst-Vm situation in Connolly \& Jones, 1970), but not in others (e.g., Millar, 1972). It was suggested that errors are smaller in the Kst-Vm design only if visual cues are available during kinesthetic standard presentation (Newell, Shapiro, \& Carlton, 1979).

Actual engagement of any modality in perception of standard was avoided in Experiment 1 in order to get around 


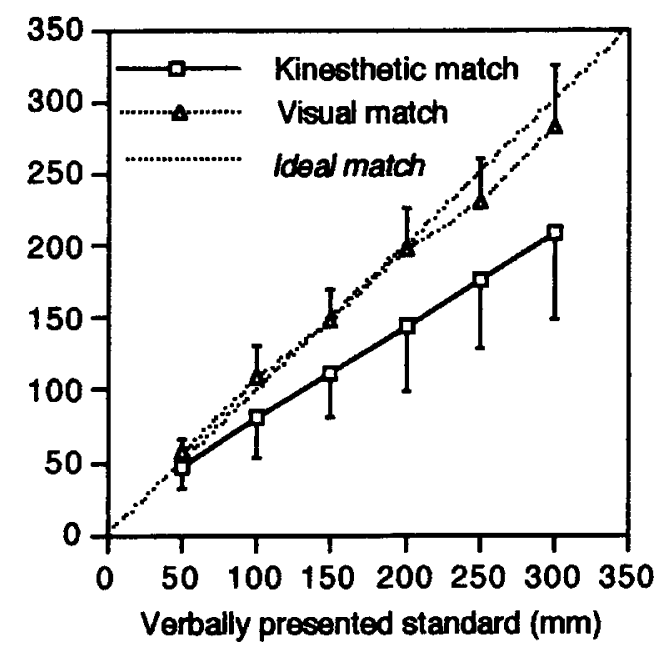

Figure 1. Mean matching lengths and standard deviations as a function of standard length and response modality in Experiment 1.

the problem of asymmetry and other possible problems associated with the standard presentation. The standard length (in centimeters) was given verbally instead of perceptually, and the responses were visual and kinesthetic. Since the numbers in Experiment 1 represented centimeters, they had constant meaning for each subject, allowing comparisons to be made between visual and kinesthetic responses to the same numbers. Therefore, a method of magnitude production was used to explore the relationship between the two modalities (two sensation scales), instead of the relationship between the subject's sensation/number scale and the physical scale, as usual.

\section{Method}

Subjects. Fourteen students, 15-18 years of age, participated in the experiment. They all attended a psychology course at the educational institution for the gifted at the Youth Research Center Petnica.

Material. Visual responses were given on a $40 \times 60 \mathrm{~cm}$ paper, on which a horizontal line of $50 \mathrm{~cm}$ was drawn. Paper of the same dimensions, a short penc1 ( $3-4 \mathrm{~cm}$ long), and opaque glasses were used for the kinesthetic responses.

Design and Procedure. Each subject had 36 trials: 6 (5-, 10-, $15-, 20-, 25-$, and $30-\mathrm{cm}$ standard lengths) $\times 2$ (response modalities) $\times 3$ (trials). Half of the subjects performed the visual block first, and the other half performed the kinesthetic block first. Both blocks were done during one session. The assignment of a standard was the same for both trial blocks: The experimenter verbally assigned lengths one by one, in quasi-random order.

Kinesthetic responses. The subjects drew lines without visual control. A short pencil was used to keep the distance from the hand to the paper constant. The subjects wore opaque glasses that completely occluded their visual field.

Visual responses. The subjects marked off the desired length on the existing line, beginning from its left end. After each trial, the experimenter measured the marked length and completely erased the pencil trace off the paper.

In the instructions, the subjects were told that lengths of $5-30 \mathrm{~cm}$ would be assigned to them one by one in random order and that they should mark off the specified length on the paper (visual block) or draw the line of the appropriate length (kinesthetic block). The orientation in which the lines should be drawn was not specified, but all the subjects drew lines within a $0^{\circ}-45^{\circ}$ inclination from the horizontal.

\section{Results and Discussion}

The data were mean response lengths in millimeters. The means were based on 3 trials per length per subject. The factors in $2 \times 6$ analysis of variance (ANOVA) were standard length $(5,10,15,20,25$, and $30 \mathrm{~cm})$ and response modality (visual or kinesthetic). The results are shown in Figure 1.

Effect of length. The main effect of length was significant $[F(5,60)=270.37, p<.001]$. The response length increased with the standard length.

Effect of modality. The response modality had a significant effect $[F(1,2)=38.87, p<.001]$. Kinesthetic responses were $19 \%-27 \%$ shorter than the visual responses, depending on the standard length. The interaction between modality and length was significant $[F(5,60)=$ 14.44, $p<.001$ ]. As can be seen in Figure 1, the size of the difference between the modalities increased with the standard length.

Variability of the responses. Standard deviations were larger for longer response lengths. They reflect individual differences, since each subject contributed a single score to the average response for each standard length.

The variability of the kinesthetic responses was larger than the variability of the visual responses. In Experiment 1, the standard was not perceptually available. However, perhaps, implicitly, the standard was visual, a visual representation of length. If this was so, kinesthetic responses resulted from intermodal comparisons (visual representationkinesthetic response), whereas visual responses resulted from intramodal comparisons (visual representationvisual response). This is relevant, because the variability of matches in intermodal matching experiments is larger than the variability of matches in intramodal experiments (Connolly \& Jones, 1970; Millar, 1972). Alternatively, a larger kinesthetic variability may reflect lower precision of this modality.

Best-fitting function. The data for this calculation were 6 mean visual responses (V) and 6 mean kinesthetic responses $(K)$ in millimeters, one for each standard length. Restricting ourselves to the description of the range of data obtained, linear function would be the simplest bestfitting function ( $r^{2}$ for the linear fit for this set of data was .997). However, as pointed out by a referee, intercepts of linear functions calculated were all different from zero, predicting that when the kinesthetic size is zero, the visual is not (or vice versa). Therefore, if we want to extrapolate on the basis of the results, and with the constraint that function has to go through the beginning of the coordinate system, power function fits better. However, it is an extrapolation, and there is a possibility that the whole range of data cannot be described by a single function.

The best-fitting power function, equation, and $r^{2}$ are presented in Figure 2 (computer application Cricket Graph III was used to calculate this and all other best-fitting functions in the study). The line is almost straight. When the kines- 


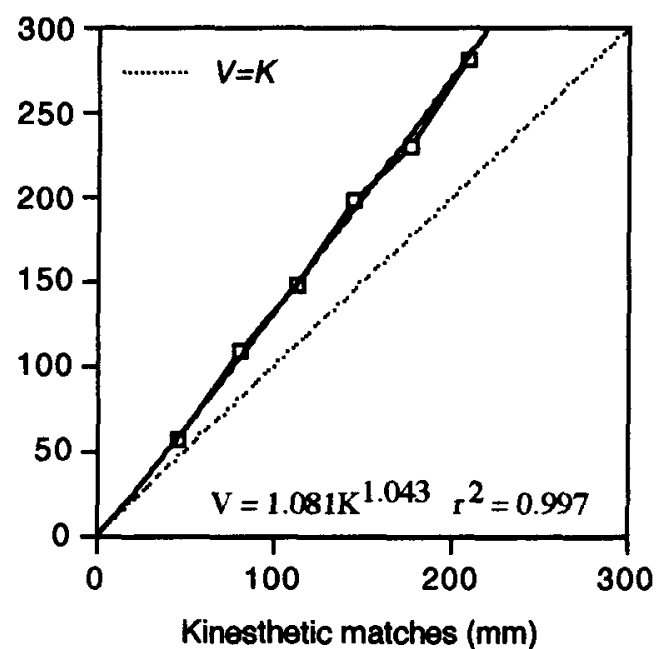

Figure 2. Power function relating apparently equal kinesthetic and visual lengths in Experiment 1.

thetic length, which is arbitrarily chosen to be a predicting variable, is doubled, the visual length grows by factor 2.06 .

The results of Experiment 1 were opposite to the results of a similar experiment performed by Jastrow (1886). In one of his experiments, the lengths were assigned verbally, and the subjects either visually chose the line of the appropriate length or expressed the length by a hand movement. The visual choice was about $10 \%$ shorter than the standard length, and the kinesthetic response about $20 \%$ longer. Unfortunately, Jastrow did not specify which lengths he assigned.

Jastrow (1886) proposes that the visual responses were given on the basis of the visual representation of length, and kinesthetic responses were given on the basis of kinesthetic representation. The alternative is that both visual and kinesthetic responses are given on the basis of a single modality representation, probably visual. Whichever is the true, differences between responses show that vision and kinesthesia differ in regard to length perception.

In summary, the main findings of Experiment 1 were (1) all kinesthetic responses were shorter than visual responses, the size of the difference depending on the length and not reaching significance for the shortest length, (2) individual differences increased with length, and (3) kinesthetic responses were more variable than visual responses.

\section{EXPERIMENT 2}

In Experiment 2, the standard was a visually presented rod in a frontoparallel plane. Visual and kinesthetic matches were given in three orientations: frontoparallel, medial, and oblique.

The orientation was varied because intramodal size percepts within both modalities depend on it. In vision, lines with vertical orientation are apparently longer than equal horizontal lines. (To be precise, according to Avery \& Day, 1969, a longer line does not have to be vertical either physically or apparently; the line corresponds to a certain retinal meridian, and the terms "vertical" and "horizontal" are misleading. However, these terms are appropriate if the observer is in the usual upright position.) Therefore, visual matches in the medial plane (vertical retinal orientation) should be shorter than visual matches in the frontoparallel plane (horizontal retinal orientation) for the same standard. Comparable orientation dependence has been found in kinesthesia: Straight movement in the medial plane appears longer than the movement of the same length in the frontoparallel plane (Cheng, 1968; Wong, 1977). Therefore, kinesthetic matches should also be shorter in the medial than in the frontoparallel plane. If two modalities are equally affected by orientation, the relationship between them was expected to remain the same in all three orientations in Experiment 2; otherwise, it was expected to vary with orientation.

The purposes of this experiment were (1) to establish the relationship between visual and kinesthetic matches over the range of lengths from 7 to $40 \mathrm{~cm}$ when the standard is visual and (2) to see whether this relationship varies with the orientation of the matches.

\section{Method}

Subjects. Sixteen first-year undergraduates from the Department of Psychology, University of Belgrade, participated in the experiment, fulfilling their course requirement.

Material and Apparatus. The visual standard was a rod horizontally placed on a $17.5-\mathrm{cm}$-high stand. The middle of the rod was $1 \mathrm{~m}$ away from the subject's eyes. Four rods were used: $7,18,29$, and $40 \mathrm{~cm}$ long, with an $8 \times 8 \mathrm{~mm}$ cross section. The subjects were also shown another four rods $(5,15,35$, and $50 \mathrm{~cm})$, "false" standards, in order to prevent them from learning the original four lengths. Matches to the latter four rods were not analyzed.

The apparatus (Figure 3) was constructed for several purposes: (1) it served as a hand guide for kinesthetic matches, (2) visual matches were also produced on it, and (3) it was a measuring device for both kinds of matching lengths. The apparatus consisted of three wooden bars: one in a frontoparallel plane, one in the medial, and the third was splitting the angle between them in two.

A groove was cut into each bar $(0.3 \times 1.2 \times 57.5 \mathrm{~cm})$. There was a movable stopper on each axis, which could be placed at any distance from the beginning of the groove up to $50 \mathrm{~cm}$. The starting position of the stopper in each experiment was about $50 \mathrm{~cm}$ from the center. There was a ruler on the side of each axis, invisible to the subjects, which showed the distance of the stopper from the center. The apparatus was placed flat on the table in front of the subject.

During the kinesthetic matching tasks, the subjects wore a thimble in order to prevent the use of tactual information while they judged the length of the groove, and they wore opaque glasses to prevent them from seeing the apparatus.

Design and Procedure. Each subject had 88 trials: 4 (standard sizes) $\times 3$ (matching orientations) $\times 2$ (matching modalities) $\times 3$ (trials) + 16 ("false" standards). The experiment consisted of two trial blocks (visual and kinesthetic) made up of 44 trials, and they were performed in separate sessions. There was a pause of a few minutes within each block and a 1- or 2-day pause between blocks. The order of trials within blocks was random, and the order of blocks was balanced. Each block was preceded by a practice trial. 


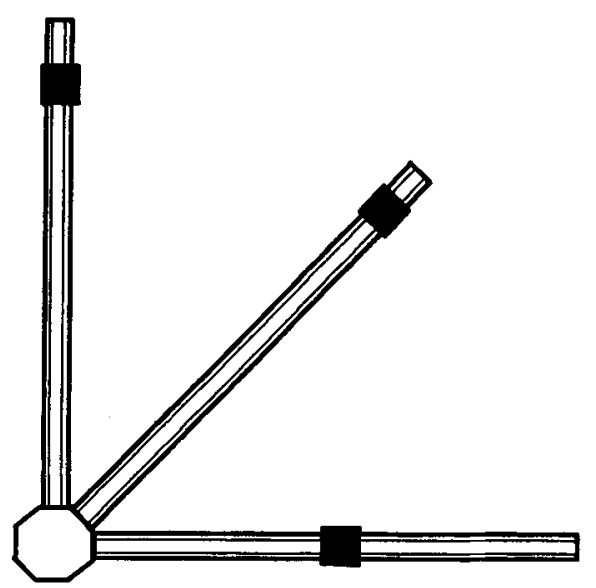

Figure 3. Response apparatus in Experiment 2. The apparatus was flat on the table in front of the subject, with its origin placed under the subject's chin. Dark squares are the stoppers adjustable to any position along the bars. For other details, see text.

The subjects were instructed to pay attention to the length of the standard rod and to adjust the length of the groove by using the stopper so that the rod could exactly fit into the groove (i.e., to make the groove as long as the rod). Action (putting the rod into the groove) was implicated in the instructions so that (1) in the context of action, the perceptual task would make more sense to the subjects (see, e.g., Michaels \& Carello, 1981) and (2) the instructions would be "objective" (i.e., having the subjects focus their attention on objectsthe rod and the groove-and not on their percepts of length). These two kinds of instructions (apparent vs. objective) could result in different judgments (M. Teghtsoonian, 1965).

The standard was present all of the time, and the response time was not limited. Other details of the procedure differed depending on the matching modality.

Kinesthetic matches. The subjects wore opaque glasses, and they sat with their heads slightly inclined. The glasses were placed close to the tip of the nose, so they could not see any part of the apparatus that was in front of them, but they could easily see the standard rod, which was about $1 \mathrm{~m}$ away and raised on the stand. The glasses were covered with paper that extended below their lower edge. This made it impossible for the subjects to see anything in the lower part of their visual field, even if they raised their heads all the way up. Therefore, the only way for the subjects to see the apparatus that was in front of them (which they were not supposed to see) was to make considerable movement downward, which could not go unnoticed by the experimenter

The subjects were instructed to make the first movement trying to approximate the length of the rod. They moved the index finger (with the thimble on it) along the groove, from the center of the apparatus outward, and when they stopped, the experimenter put the stopper next to the finger, marking the distance the finger passed. The subjects were then allowed to make as many movements as they wanted in both directions. They were watching the standard all the time. If they felt that the groove was longer or shorter than the standard rod, they could change the position of the stopper, and they could do it as many times as needed. When the length of the groove finally appeared to them to be equal to the length of the rod, the experimenter read and recorded the selected distance, without providing feedback.

Visual matches. The subjects alternately watched the standard rod and the apparatus, and they adjusted the position of the stopper until they were satisfied. They moved the stopper themselves, but this movement was irrelevant to the perception of the length of the groove. (Recall that the starting position of the stopper in each trial was about $50 \mathrm{~cm}$ away from the starting point, the origin of the grocve. This means that, for example, in order to adjust the stopper to a distance of $40 \mathrm{~cm}$, the subject had to move it $10 \mathrm{~cm}$.)

\section{Results and Discussion}

The design of this experiment allowed for three different comparisons: between the standard and each of the matches, and between visual and kinesthetic matches. The latter is methodologically superior, because it controls for biases, such as the regression toward the mean, assuming that it equally affects responses of both modalities.

Differences between visual and kinesthetic matches as a function of standard length and orientation of the match. Data for the ANOVA were lengths of visual and kinesthetic matches (lengths of the grooves as adjusted by subjects) in millimeters. The factors for the $4 \times 3 \times 2$ ANOVA were standard length $(70,180,290$, and $400 \mathrm{~mm})$, orientation of the match (frontoparallel, oblique, and medial), and matching modality (visual and kinesthetic). The results are shown in Table 2 and in Figures 4 and 5.

Effect of length. The main effect of length was significant-the matching length increased with the standard length $[F(3,42)=1,035.89, p<.01]$.

Effect of orientation. The main effect of orientation was significant $[F(2,28)=8.05, p<.01]$, as is shown in Figure 4 . Length and orientation interacted significantly $[F=(6,84)=2.95, p<.05]$. The frontoparallel matches were significantly longer than medial for $180 \mathrm{~mm}[F(1,96)=$ $23.7, p<.001], 290 \mathrm{~mm}[F(1,96)=5.16, p<.05]$, and $400 \mathrm{~mm}[F(1,96)=0.36, p<.01]$, and they were close to the lowest level of significance for $70 \mathrm{~mm}[F(1,96)=4.27, p<$ $.055]$. The difference between frontoparallel and oblique orientation was significant only for $180 \mathrm{~mm}$, with the frontoparallel match being longer $[F(1,96)=7.47, p<.01]$. Oblique matches were significantly longer than medial for $180 \mathrm{~mm}[F(1,96)=4.56, p<.05], 290 \mathrm{~mm}[F(1,96)=$ $4.98, p<.05]$, and $400 \mathrm{~mm}[F(1,96)=5.07, p<.05]$.

Longer visual matches in frontoparallel orientation than in medial orientation reflect the vertical-horizontal

Table 2

Mean Matching Lengths (in Millimeters) as a

Function of Standard Length, Matching Modality, and Orientation of the Match in Experiment 2

\begin{tabular}{llllcllccc}
\hline & \multicolumn{3}{c}{ Kinesthesia } & & \multicolumn{3}{c}{ Vision } \\
\cline { 2 - 7 } \cline { 7 - 9 } & 70 & 180 & 290 & 400 & & 70 & 180 & 290 & 400 \\
\hline Frontoparallel & 85.3 & 173.3 & 241.3 & 321.5 & & 72.2 & 180.7 & 269.9 & 378.5 \\
Oblique & 86.1 & 167 & 245.3 & 327.3 & & 71.4 & 168.9 & 268.1 & 375.6 \\
Median & 82 & 157.5 & 239 & 321.1 & & 70.7 & 163.5 & 257.8 & 371.1 \\
\hline
\end{tabular}




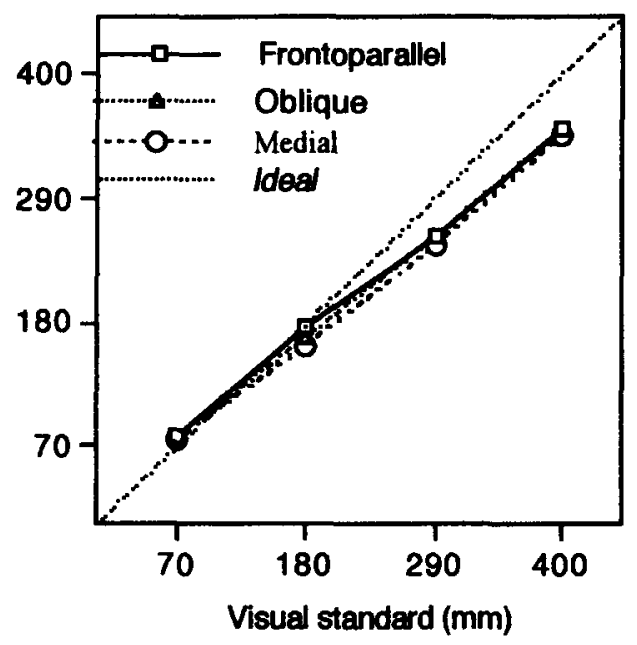

Figure 4: Mean matching lengths as a function of standard length and orientation of the match in Experiment 2. Standard deviations for the 70-, 180-, 290-, and 400-mm standards were 14, 24,35 , and $51 \mathrm{~mm}$, respectively, in the frontoparallel condition, $15,24,37$, and $55 \mathrm{~mm}$, respectively, in the oblique, and $13,22,38$, and $50 \mathrm{~mm}$, respectively, in the medial.

illusion. The illusion is effective with the oblique lines as well, making them appear shorter than vertical lines and longer than frontoparallel lines (Coren \& Girgus, 1978). Most of the differences between vertical and horizontal orientation were small: $2 \%, 10 \%, 4 \%$, and $2 \%$ for the lengths of $7,18,29$, and $40 \mathrm{~cm}$, respectively. According to other studies, strength of the vertical-horizontal illusion is proportional to the length of the stimuli. For example, according to Kuennapas (1955), vertical lines appear to be $7 \%$ to $14 \%$ longer than horizontal lines. The reason for this discrepancy was probably the foreshortening of the line presented in a medial plane on a table surface in this experiment, which does not occur when both lines are presented in the frontoparallel plane, as is usually the case.

Kinesthetic matches were also larger in the frontoparallel orientation than in the medial orientation, which means that they appeared shorter. This finding is in accord with the results of several studies (e.g., Cheng, 1968; Wong, 1977). However, the average difference between orientations obtained in the other studies was about $10 \%$ (Loomis \& Lederman, 1986), whereas the present difference was not proportional to the length, and it almost disappeared for the longer standards: $4 \%, 9 \%, 1 \%$, and $0 \%$ for the $7,18,29$, and $40 \mathrm{~cm}$, respectively.

The strongest effect of orientation, and the only one comparable in size to the effects usually obtained in other studies, was obtained for the $18-\mathrm{cm}$ standard length for both matching modalities.

Effect of modality. The main effect of the matching modality was significant $[F(1,14)=6.38, p<.05]$. The modality and length interaction was also significant $[F(3,42)=21.54, p<.001]$. However, there was no significant interaction between modality and orientation, showing that the intermodal relationship was similar in all orientations. The interaction among modality, orientation, and length was also not significant.

For the shortest standard, kinesthetic matches were $16 \%-18 \%$ longer than visual matches $[F(1,42)=4.79, p<$ $.05]$. For longer lengths, the reverse was true: Visual matches were longer than kinesthetic matches, and the difference grew with the standard length. For the $180-\mathrm{mm}$ standard, the difference was $1 \%-4 \%$, and it was not significant $[F(1,42)=0.74]$. For $290 \mathrm{~mm}$, the difference was $7 \%-11 \%$ $[F(1,42)=15.45, p<.001]$, and for $400 \mathrm{~mm}$, the difference was $13 \%-15 \%[F(1,42)=75.54, p<.001]$, depending on the orientation of the standard (see Table 2 and Figure 5).

Variability of the matches. Standard deviations were larger for longer standards and matches (Figures 4 and 5). As in Experiment 1, they represent individual differences.

The variability for both modalities was smaller than in Experiment 1 (compare Figure 5 and Figure 1). Smaller variability in Experiment 2 was probably due to the visual standard presentation as opposed to the verbal assignments in Experiment 1. In Experiment 1, the subjects had to imagine lengths, whereas, in Experiment 2, lengths were physically present, and, therefore, between-subjects variability in their apparent size should be smaller.

Standard deviations for the kinesthetic matches were larger than for the visual matches. The visual matches were the result of intramodal matching (the standard was visual), which results in smaller variability than does intermodal matching (Connolly \& Jones, 1970).

Best-fitting function. The data for this calculation were four mean visual responses (V) and four mean kinesthetic responses $(K)$ in millimeters, one for each standard length. Best-fitting power function, equation, and $r^{2}$ are presented in Figure 6. The imaginary ideal correspondence is shown by the dotted line $(\mathrm{V}=\mathrm{K})$. When the kinesthetic length was doubled, visual length grew by factor 2.36 .

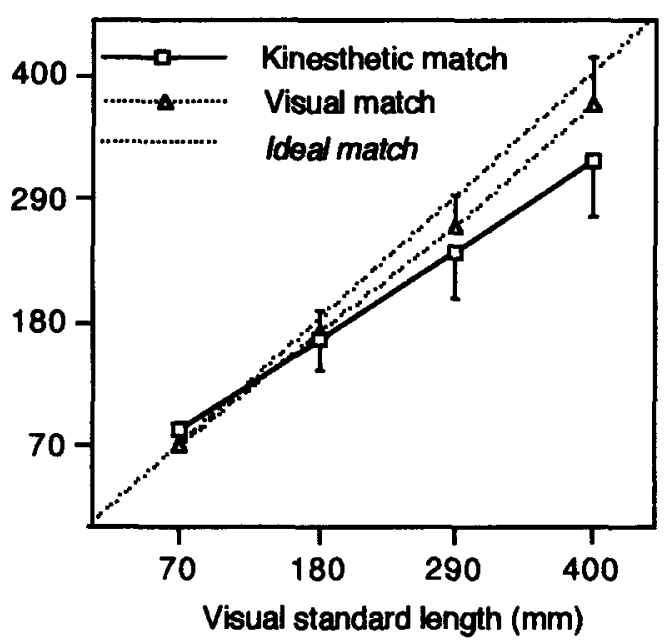

Figure 5. Mean matching lengths as a function of standard length and response modality in Experiment 2 . Standard deviations for the 70-, 180-, 290-, and 400-mm standards were 16, 28, 40, and $49 \mathrm{~mm}$, respectively, for kinesthesia, and $7,19,28$, and $39 \mathrm{~mm}$, respectively, for vision. 


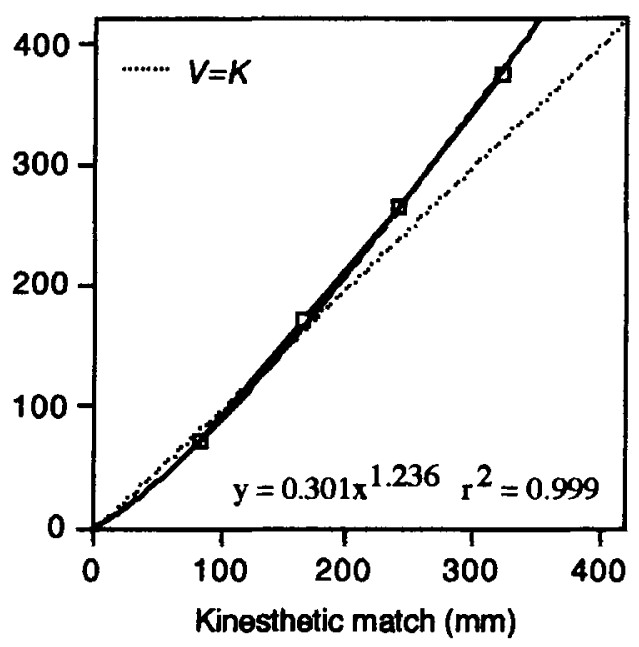

Figure 6. Power function relating apparently equal kinesthetic and visual lengths in Experiment 2.

To conclude, in Experiment 2, significant mismatch was found between apparently equal lengths perceived by vision and by kinesthesia. The size and direction of the difference depended on length. The size of the mismatch did not vary with the orientation of matches. The strength of the effect of orientation was similar for both modalities, and it depended on the length.

\section{EXPERIMENT 3}

Research on intermodal perception of size has dealt mainly with the perception of linear extents (i.e., straight arm movements and lines presented to vision; for references, see Table 1, and Hatwell, 1990). However, a few recent studies have shown that vision and kinesthesia also differ in perception of circular sizes. Laszlo et al. (1994) moved their subjects' arms passively around a circle $15 \mathrm{~cm}$ in diameter. The subjects then matched this kinesthetically perceived circle by choosing among the visually presented circles, and, on average, they overestimated it by $37 \%$.

In previous research, I (Seizova, 1995) compared the circles perceived visually and circles drawn without visual guidance (active movement). The circles drawn and not seen were, on average, smaller than the visually perceived ones (however, since the figures drawn without visual guidance were generally irregular, some doubts concerning the size of the difference between apparently equal visual and kinesthetic "circles" remained). A similar result with different procedures was obtained by Zelaznik and Lantero (1996): When the visual control of the hand during repetitive circular movements (about $12 \mathrm{~cm}$ in diameter) was withdrawn, and only the kinesthetic was left, the circles became significantly smaller in size.

Circles of different sizes were used in Experiment 3. Instead of drawing, the subjects moved the index finger along the circular grooves (rings), and the problems of measurement of irregular drawings were avoided. The standards were visually perceived rings, and the subjects responded by choosing an appropriate ring from a series felt by kinesthesia.

The purpose of Experiment 3 was to establish the difference between modalities as a function of the size of rings.

\section{Method}

Subjects. Eighteen first-year undergraduates from the Department of Psychology, University of Belgrade, participated in the experiment. Eleven of them had participated in Experiment 2.

Material. Four cardboard rings were used as standards. Each was $10 \mathrm{~mm}$ wide. Circumferences of the midlines (middle circles) of the rings were $176,229,283$, and $336 \mathrm{~mm}$, and the corresponding radii were $28,36,45$, and $53 \mathrm{~mm}$. Eighteen rings that served as the matching set were cut into the first layer of the thick, two-layered cardboard (Figure 7). The cardboard was $92 \mathrm{~cm}$ long, $5 \mathrm{~cm}$ wide on the one side, and $16 \mathrm{~cm}$ on the other. The difference between midlines of the rings was originally planned to be constant, $18 \mathrm{~mm}$, but it varied from 16 to $21 \mathrm{~mm}$ due to imprecision in the production of the material. The range of the circumferences was $97-408 \mathrm{~mm}$. Rings in the matching set were numbered 1-18, with the smallest one carrying number 1 . The four standard rings were equal in size to Rings 5, 8,11 , and 14 .

Apart from the standard rings, the subjects were presented with several "false" standard rings, in order to increase the variety of standards and to prevent the subjects from memorizing them. The matches to these rings were not analyzed.

Design and Procedure. Each subject made 36 matches: 4 (standard sizes) $\times 2$ (matching modalities) $\times 3$ (trials) +12 ("false"standards). Visual and kinesthetic trials were separated into two blocks, and they were both done in one session. The order of trials within each block was quasi-random, and the order of blocks was balanced. Each block was preceded by a practice trial.

The procedure was similar to the procedure in Experiment 2: Visual standard (a ring) was presented at a distance of about $1 \mathrm{~m}$ from the subject's eyes, and it was present all of the time that the subject was searching for an appropriate matching ring. The subjects were told to pay attention to the size of the ring and to find the matching ring groove in front of them that the standard ring could fit in (i.e., to find the ring of the same size as the standard). Depending on the matching modality, the procedures were as follows.

Kinesthetic matches. As in Experiment 2, the subjects wore opaque glasses lowered toward the tip of the nose, and they could only see the standard ring, not the cardboard with the matching rings. Using the index finger (with the thimble on it), they would find the groove that was placed in front of them. They were encouraged to slide the finger around the ring as long as they needed to get an idea of the ring size.

The movement direction was not defined, and the subjects often changed it while inspecting the ring. After inspecting one ring, they went to the next one, elther a smaller or a larger ring in the series, and they continued to do so until they found the ring that was apparently equal to the standard. Either alone or with the help of the experimenter, the subjects moved the cardboard to the left or to the right in order to put the ring they were investigating in front of themselves. In the beginning of each trial, the middle part of the cardboard was placed in front of the subject, or it remained in the position left after the previous trial.

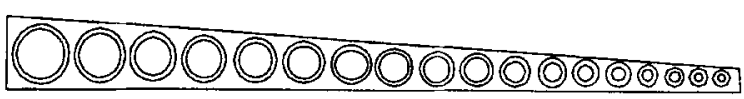

Figure 7. Matching-rings set. 


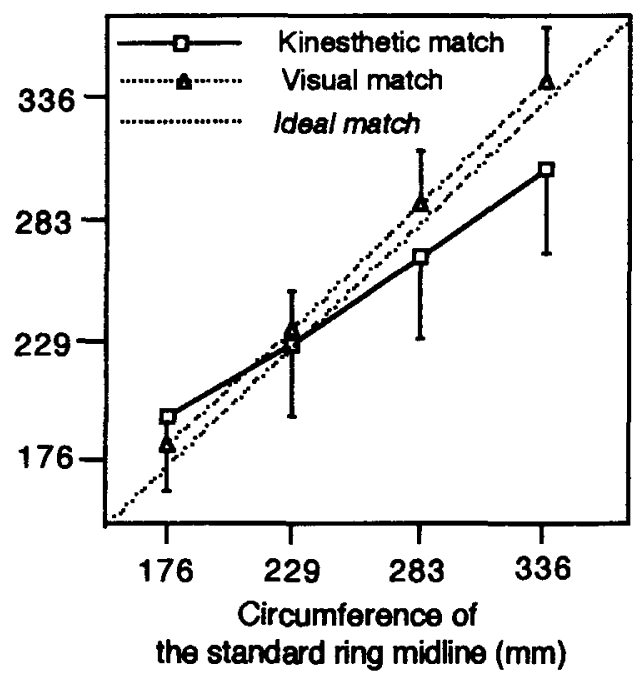

Figure 8. Mean size of the matching rings and standard deviations as a function of standard size and matching modality.

The subjects were always encouraged to inspect the ring in the set next to the ring they were about to choose and to make a final decision afterwards. The purpose was to avoid or reduce the effect of the tendency to choose the first ring similar to the standard ring. The subjects did not touch the matching rings with the other fingers or with the other hand.

Visual matches. The subjects alternately inspected the standard ring and the matching set, and they visually chose the appropriate ring. They were encouraged to place the matching ring they were inspecting in front of them by moving the cardboard to the appropriate position. Therefore, the final decision always concerned the matching ring looked at in that position. The distance from the matching ring to the subject's eyes was approximately $35 \mathrm{~cm}$.

\section{Results and Discussion}

Data for a $4 \times 2$ ANOVA were mean serial numbers of the rings calculated on the basis of three trials that each subject had per situation. The factors were the standard ring size (rings $5,8,11$, and 14 with mean circumferences of 176, 229, 283, and $336 \mathrm{~mm}$, respectively) and matching modality (vision and kinesthesia). The results are shown in Figure 8 (note that, instead of the serial numbers of the rings, the approximate middle circumferences of these rings are shown on both axes of the graph).

Only the standard size had a significant main effect $[F(3,48)=588.47, p<.001]$. The interaction between the standard size and the matching modality was also significant $[F(3,48)=65.53, p<.001]$, and the differences between the modalities for each of the standard sizes were significant. Visual matches were larger than kinesthetic matches for all standard sizes except the smallest one, for which the reverse was true. For the $176-\mathrm{mm}$ standard, $F(1,48)=23.47, p<.001$; for the $229-\mathrm{mm}$ standard, $F(1,48)=5.26, p<.05$; for the 283 -mm standard, $F(1,48)=$ $67.82, p<.001$; for the 336-mm standard, $F(1,48)=197.41$, $p<.001$.

Variability of the matches. As in Experiments 1 and 2 , standard deviations reflect individual differences; they were larger for larger sizes, and they are larger for kinesthesia than for vision.

Best-fitting function. The data for this calculation were four mean visual and four mean kinesthetic matchingrings circumferences in millimeters, one for each standard length. Best-fitting power function, equation, and $r^{2}$ are presented in Figure 9. The imaginary ideal correspondence is shown by the dotted line $(\mathrm{V}=\mathrm{K})$. When the kinesthetic circumference was doubled, visual grew by a factor of 2.68 .

The main findings of Experiment 3 are the following: As in Experiments 1 and 2, apparently equal sizes perceived by vision and by kinesthesia were significantly different, and the size and direction of the difference depended on the size perceived. The sign of the difference reversed for the smallest standard ring, relative to the larger ones. This had not been observed before. For larger circles, visual size was larger than kinesthetic size, which is in accordance with the results of Laszlo et al. (1994), Seizova (1995), and Zelaznik and Lantero (1996).

Individual differences in Experiments 2 and 3. Out of the 16 subjects who participated in Experiment 2, 11 participated in Experiment 3. In both experiments, the standard was visual, and the matches were visual and kinesthetic. The shape of the standard was different: linear in Experiment 2, and circular in Experiment 3. Individual results were analyzed to see if there were consistent individual differences across experiments. Two results were found:

(1) The absolute size of the responses differed. Some subjects tended to give larger responses, and some tended to give smaller ones. The coefficients of linear correlation between the responses in the two experiments were significant both for vision $[r=.742, F(1,9)=11.031, p<.01]$ and for kinesthesia $[r=.607, F(1,9)=5.254, p<.05]$.

(2) The size of the intermodal mismatch differed. Some subjects had larger differences between visual and kinesthetic matches than did others. The difference between the

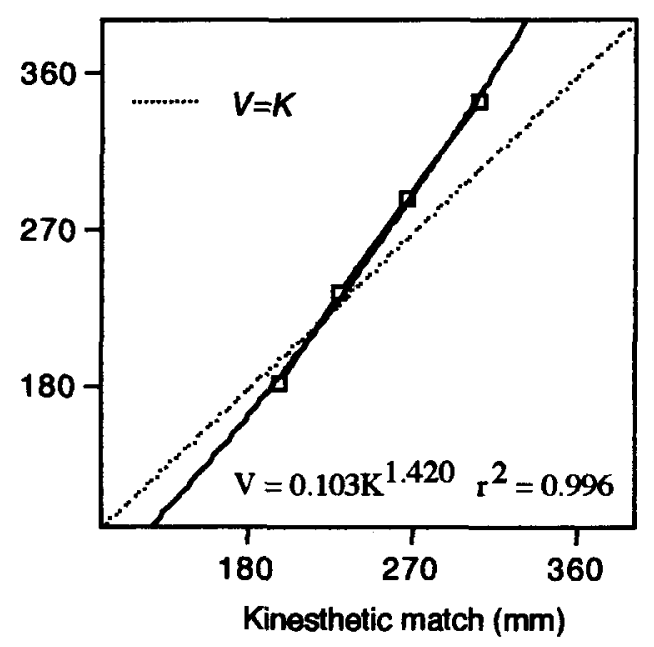

Figure 9. Power function relating apparently equal kinesthetic and visual sizes in Experiment 3. 
kinesthetic and visual responses is expressed as the ratio between them ( $\mathrm{K} / \mathrm{V}$ ratio). The correlation between the $\mathrm{K} / \mathrm{V}$ ratios from Experiments 2 and 3 for 11 subjects was significant $[r=.681, F(1,9)=7.77, p<.05]$.

The tendency to give smaller or larger responses may have been due to the perceptual differences between subjects (they all perceived these responses as equal to the standard) or the nonperceptual differences (e.g., the tendency to exaggerate or to be cautious). However, it is likely that the size of the difference between the visual and kinesthetic responses has a perceptual origin. The alternative is that nonperceptual factors affect the modalities differently, which we have no reason to assume.

\section{EXPERIMENT 4}

In order to describe intermodal relations in length perception, it is essential to know intramodal relations, both visual and kinesthetic variations. Visual illusions have attracted more interest (see, e.g., a review by Coren \& Girgus, 1978). Probably not so well known is the fact that size illusions also exist in kinesthesia. Movement length perception depends on the orientation of movement. If the movement is performed on a table surface, the straight movement in the medial plane appears longer than the movement of the same length in the frontoparallel plane (Cheng, 1968; Wong, 1977). Apparent movement length also varies with speed, slower movements being apparently longer than faster ones, both active (Ono, 1969) and passive (Paillard \& Brouchon, 1974).

In the study by Brown et al. (1948), positioning response varied with the direction of movement. The positioning response was probably not based on the length perception; therefore, their result does not necessarily imply that there is a difference in the perception of length as a function of direction of movement. Still, that is a possibility. This question was addressed in the present experiment.

In Experiment 4, the standard was kinesthetic, and the matches were visual (recall that, in Experiments 2 and 3, these were reversed). The standard movement varied in length, direction, and orientation. The variations in the size of the visual match were analyzed; they reflect the variations in the apparent movement length as a function of the movement length, direction, and orientation. The relationship between movement lengths and matching visual lengths was also analyzed.

\section{Method}

Subjects. Eighteen first-year undergraduates from the Department of Psychology, University of Belgrade, participated in the experiment.

Material. The same apparatus used in Experiment 2 was used in Experiment 4. However, the oblique axis was taken out, and only the frontoparallel and medial axes were used for the presentation of the kinesthetic standard. The apparatus was placed flat on the table in front of the subjects. The subjects slid the index finger (with thimble on) along the apparatus' grooves. Visual matches were arranged as a series of 40 rods $1-40 \mathrm{~cm}$ long, and $8 \times 8 \mathrm{~mm}$ wide. Each was $1 \mathrm{~cm}$ longer than the previous one. The rods were horizontally oriented and fixed to the cardboard $(41.5 \times 60 \mathrm{~cm})$. The shortest rod was at the top. The rods were numbered, and the subjects read the number of the rod when they chose it. The cardboard was fixed to a wall, $1 \mathrm{~m}$ away from the eyes of the subject.

Design and Procedure. The subjects performed 48 trials each: 3 (standard movements: 8,18 , and $28 \mathrm{~cm}) \times 2$ (orientations: frontoparallel and medial) $\times 2$ (directions: toward and away from the subject) $\times 3$ (trials) +12 ("false" standards). Half of the subjects performed movements toward themselves first, and the other half performed movements away from themselves first. Blocks were done in sessions separated by 1 day or 2 days. "False" trials were introduced in order to increase the variety of the standard lengths, and they were not analyzed.

At the beginning of each trial, the subject was seated with hands in the lap, looking at the set of rods on the wall. The subject wore opaque glasses lowered toward the tip of the nose, as in Experiments 2 and 3 . This prevented the subjects from seeing any part of the apparatus on which they performed their movements, but it allowed them to see the rods on the wall. The experimenter placed the stopper of the apparatus in the appropriate position (e.g., at a distance of $18 \mathrm{~cm}$ ), thereby fixing the desired length of the movement. The subjects were instructed to put the index finger in one of the grooves and to perform a movement in a specified direction (toward or away from themselves). When making the movement away from themselves, they placed the finger at the beginning of the groove; when the direction of movement was toward themselves, they placed the finger at the rear end of the groove, next to the stopper.

In the instructions, the subjects were told that they would perform three movements in each trial. The first movement was meant to give them a crude idea of the length of the groove, and the second and third movements were meant to enable them to perceive the length of the groove better and to find the rod of the same length. If they found it necessary, the subjects were allowed to make more than three movements, but only few of them asked to do this. The subjects returned their hands to the starting position by a roundabout movement, in order to prevent them from making judgments about the movement length on the basis of the movement direction opposite to the desired one. After the third movement, the subjects told the experimenter the number of the rod they had chosen.

\section{Results and Discussion}

Differences between visual matches as a function of length, orientation, and direction of the standard. The data for the $3 \times 2 \times 2$ ANOVA were visual matching lengths in millimeters. The factors in the ANOVA were standard movement length $(8,18$, and $28 \mathrm{~cm})$, standard movement orientation (frontoparallel and medial), and standard movement direction (toward oneself and away from oneself). The results are shown in the Figure 10.

Effect of length. A significant main effect of length was obtained $[F(2,34)=789.67, p<.001]$, indicating that the matching length increased with the standard length.

Effect of orientation. The main effect of orientation was significant $[F(1,17)=32.62, p<.001]$. Visual matches to the standard movements along the medial axis were longer (i.e., these movements were perceived as longer) than the visual matches to the movements along the frontoparallel axis. This finding - larger apparent length of the movements in the medial plane than in the frontoparallel plane-is in accord with the previous research (e.g., Cheng, 1968; Wong, 1977) and the findings of Experiment 2. 


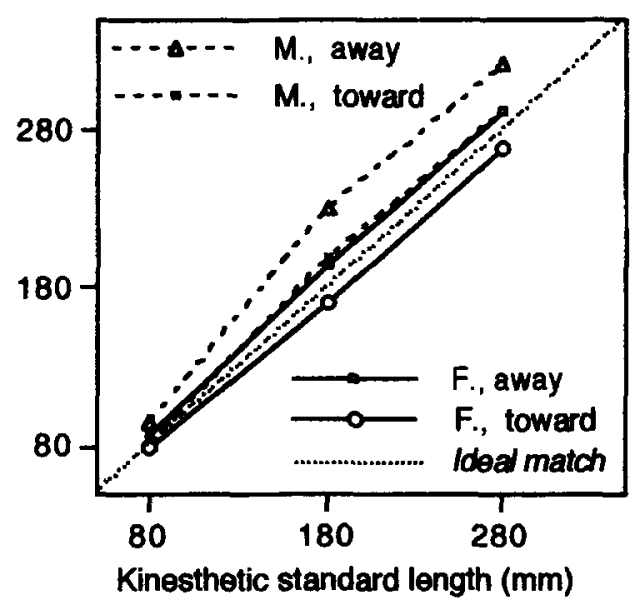

Figure 10. Mean matching lengths as a function of length, direction, and orientation of the standard movement. $M$ stands for movement in a median plane, and $F$ stands for movement in a frontoparallel plane. Standard deviations for the 80-, 180-, and $280-$ mm standards were 25,41 , and $42 \mathrm{~mm}$, respectively, for the medial movement away, 16, 37, and $41 \mathrm{~mm}$, respectively, for the medial movement toward, 14,28 , and $30 \mathrm{~mm}$, respectively, for the frontoparallel movement toward, and 19,40 , and $50 \mathrm{~mm}$, respectively, for the frontoparallel movement away.

Length and orientation interacted significantly $[F(2,34)=$ $10.77, p<.001]$, because the orientation effect was not significant $[F(1,34)=2.27, p<.141]$ for the shortest standard length $(80 \mathrm{~mm})$.

The effect of orientation of the kinesthetic matches in Experiment 2 was not as prominent as the effect of orientation of the kinesthetic standard in Experiment 4. In Experiment 2 , the medial axis appeared 1\%-9\% longer than the horizontal, depending on the length; in Experiment 4, it appeared about $9 \%$ longer for all lengths. It is not clear what caused this difference.

Effect of direction. A significant main effect was obtained for the direction of the standard movement $[F(1,117)=$ $23.95, p<.001]$. The visual match to the standard movements away from the subject was larger than that to the standard movements toward the subject, meaning that movements away from the subject's body appeared longer than movements toward the subject's body. This was true both for the movements along the frontoparallel and those along the medial axis, the interaction between the direction and orientation being nonsignificant.

The difference was about $9 \%$ for all lengths, except the 80-mm standard length, for which the difference between the two directions of movements was not significant $[F(1,34)=3.54, p<.068]$. Therefore, the length and direction interaction was also significant $[F(2,34)=4.22$, $p<.05]$. Interaction between length, orientation, and direction was not significant.

Variability of the matches. Standard deviations that reflect individual differences increased with the size of the matches, as in the previous experiments.

In conclusion, $18-\mathrm{cm}$ and $28-\mathrm{cm}$ movements away appeared longer than movements toward, and those along the medial axis appeared longer than those along the frontoparallel axis. Therefore, medial movement away appeared to be the longest, followed by frontoparallel movement away and medial movement toward, which were similar; frontoparallel movement toward appeared to be the shortest. Movements of $80 \mathrm{~mm}$ were apparently the same in all conditions.

The weak point of this experiment was lack of control for the speed of movement, which may be the confounding variable. Consider the possibility that movements away are spontaneously performed at a lower speed than are movements toward. Slower movements being apparently longer (Ono, 1969), the obtained effect could be explained as an effect of the speed, instead of the direction, of movements.

Differences between standard and matching lengths. As shown by $t$ tests for the difference between theoretical and empirical mean, there were only 3 significant differences out of 12 comparisons: medial movement away of $18 \mathrm{~cm}[t(17)=5.22, p<.001]$ and of $28 \mathrm{~cm}[t(17)=4.22$, $p<.001]$ and medial movement toward of $18 \mathrm{~cm}[t(17)=$ $2.17, p<.05$ ] were all overestimated by their visual matches. There were no significant differences between visual matches and movements in other conditions.

The absence of differences between $28 \mathrm{~cm}$ perceived visually and kinesthetically in three of four conditions is at odds with the results of Experiments 1 and 2. For approximately the same lengths in the latter experiments, visual matches were larger than kinesthetic ones. These three experiments differed in several respects (designs, manners of movement, and visual stimuli presentation), and, therefore, it is not possible to specify the reason for this variability at this stage.

Best-fitting function. The calculations were based on 3 kinesthetic standard lengths $(\mathrm{K})$ and four times three visual matches (V), one for each movement direction in two orientations. The following power functions were obtained: medial movement away, $\mathrm{V}=1.336 \mathrm{~K}^{0.98}, r^{2}=.993$; medial movement toward, $\mathrm{V}=0.982 \mathrm{~K}^{1.016}, r^{2}=.997$; frontoparallel movement away, $\mathrm{V}=1.207 \mathrm{~K}^{0.979}, r^{2}=1$; frontoparallel movement toward, $\mathrm{V}=1.18 \mathrm{~K}^{0.961}, r^{2}=.999$.

According to each of these functions, when the kinesthetic size was doubled, visual size grew by approximately equal proportions: $1.97,2.02,1.97$, and 1.95 , respectively.

In summary, apparent length of a kinesthetically perceived groove (expressed through visual matches) is function of length, orientation, and direction of movement. In spite of these systematic variations of visual matches, small and nonsignificant differences were found between them and the kinesthetic standard. This absence of significant differences between two modalities is in contrast to the results of the other experiments in this study.

\section{GENERAL DISCUSSION}

This paper presents the study of intermodal relationships regarding size perception. Active movements and visual stimuli of apparently the same size were compared with respect to their real sizes. A similar overall picture emerged from this study as that found in the literature re- 
Table 3

Ratios of Apparently Equal Sizes (Kinesthetic/Visual) as a Function of the Standard Size (in Centimeters)

\begin{tabular}{clllllllllll}
\hline & \multicolumn{1}{c}{ Standard Size } \\
\hline Experiment & \multicolumn{1}{c}{ Standard } & Form & $0-5$ & $6-10$ & $11-15$ & $16-20$ & $21-25$ & $26-30$ & $31-35$ & $36-40$ \\
\hline 1 & Verbal & Linear & $0.81^{*}$ & 0.73 & 0.75 & 0.72 & 0.76 & 0.74 & n.d. & n.d. \\
2 & Visual & Linear & n.d. & 1.18 & n.d. & $0.97^{*}$ & n.d. & 0.91 & n.d. & 0.86 \\
3 & Visual & Circular & n.d. & n.d. & n.d. & 1.14 & 0.96 & 0.89 & 0.85 & n.d. \\
4 & Kinesthetic: median, away & Linear & n.d. & $0.83^{*}$ & n.d. & 0.78 & n.d. & 0.87 & n.d. & n.d. \\
4 & Kinesthetic: median, toward & Linear & n.d. & $0.96^{*}$ & n.d. & 0.90 & n.d. & $0.96^{*}$ & n.d. & n.d. \\
4 & Kinesthetic: frontoparallel, away & Linear & n.d. & $0.92^{*}$ & n.d. & $0.92^{*}$ & n.d. & $0.96^{*}$ & n.d. & n.d. \\
4 & Kinesthetic: frontoparallel, toward & Linear & n.d. & $1.00^{*}$ & n.d. & $1.06^{*}$ & n.d. & $1.04^{*}$ & n.d. & n.d. \\
\hline
\end{tabular}

Note-n.d., no data in this range of lengths. *Not significantly different from 1.

view (summarized in Table 1). Constant errors exist, but not in all conditions and not always in the same direction. Data from this study, summarized in Table 3 , in which longer lengths were used than in most of the previous research, can be added to the existing evidence.

\section{Variable Mismatch}

It is clear that the size and the direction of the intermodal mismatch depend on the lengths perceived: Longer lengths tend to be underestimated by kinesthesia, relative to vision, whereas shorter lengths tend to be either overestimated or accurately matched (Experiments 1,2, and 3). A similar trend-overestimation of short distances and underestimation of long ones - on a similar range of lengths was observed by Brown et al. (1948). However, the percentages of over- and underestimations that Brown et al. obtained were much smaller, just few a percents.

There is also an important difference in design between Brown et al.'s (1948) study and this one. It is not certain whether their results can be attributed to the perception of length/distance or to the perception of location (position) of the target. The Brown et al. study was concerned with the positioning response, and the experimental setting was the following: Subjects sat in the dark; the lights would come on for $2.5 \mathrm{sec}$ to allow them to see two markers on a slider, separated by given distance. After the lights went off again, the subjects were supposed to place one marker next to the other marker, a target, by pushing it along the track.

We should consider what was really matched in the present study. In one condition in Experiment 2, and in all conditions in Experiment 4, both the rod and the groove were in a frontoparallel plane. The subjects were supposed to feel the length of the groove by running the index finger along it. One of the strategies that the subjects could use to perform the task was to try to match the position of the finger at the beginning and at the end of the movement with the position of the left and the right ends of the rod, respectively. This was exactly the strategy reported in a recent study using a similar task, in which the strategies were systematically monitored (Seizova \& Stankov, 1998). About $20 \%$ of 104 subjects used it to solve the lengthmatching task for lengths of 40 and $50 \mathrm{~cm}$. However, the position was not always an available cue in this study: when the orientation of the standard rod and matching groove was not the same (the remaining conditions in Ex- periment 2), and when there was no standard object at all (Experiment 1) but only the notion of length in centimeters. Therefore, at least in some conditions in this study, the length was attended to.

We do not know yet what attending to length versus attending to position(s) may mean in terms of the outcome of the length-matching tasks. In order to find out, and to understand the mechanism of intermodal perception, we have to recognize which tasks allow for which of these perceptual strategies.

The study of perception of circular extents (Experiment 3) can be compared to the studies of Laszlo et al. (1994), Seizova (1995), and Zelaznik and Lantero (1996). The overestimation of kinesthetic, relative to visual, sizes appeared in all these studies. It was obtained in various conditions in the Laszlo et al. study with passive movements and with active movements (drawing) in the other two studies. It was confirmed in the present study, with active hand movements. What is novel is that the direction of the mismatch varied with size, and, for the circle of the shortest circumference, the sign of the error reversed.

The question analogous to the one asked about linear extents applies to this task: What was really perceived - the extent of the circumference of a ring, or curvature, or the size of the area? It is possible that the subjects could have been matching the curvature rather than length of the circumference of the ring. It is also possible that they were imagining the area of the ring and basing their matches on that. We cannot tell, on the basis of this study, because these measures are all necessarily interrelated.

The largest intermodal mismatch in this study was obtained with the verbal standard. When the standard was presented verbally, the subjects did not have standard objects but had only their own representations to focus on, whereas when giving a drawing response, they did not have specific objects to judge but rather their sensations/ percepts. However, in other experiments in this study, they were encouraged to make a "stimulus error" (i.e., to try to estimate the size of objects), and they probably tended to use more environmental cues. These two different directions (apparent vs. objective) may affect the results, as suggested by R. Teghtsoonian (1981).

Generally, the smallest intermodal differences were obtained in Experiment 4, with the kinesthetic standard. It is not clear why. 
The results of Experiment 4 supply evidence relevant for the question raised by Raffel (1936), which was not directly addressed in this study: What is the role of rate of stimuli presentation on the perception of size? Even the fastest movement takes more time than a glance of the line, and that may be the crucial difference between kinesthetic and visual information about length. However, varying the speed of movement did not significantly affect the size of intermodal mismatch, and Raffel concluded that the rate of stimuli presentation cannot be the single cause of the differences between visual and tactual-kinesthetic judgments. The results of Laszlo and Broderick's (1985) study support this conclusion, as well as the results of Experiment 4 . If the time was crucial, the visual display should be perceived as different from movements of the same length, and, in most conditions in that experiment, the differences were absent.

The results of this study, and the existence of constant errors generally, bear on the much-discussed issue of the origin and development of space perception (see, e.g., Marks, 1978 , ch. 2). Whatever is the cause of the constant errors, it can hardly be experience; it is hard to imagine how experience would provide us with different "sense meters" for the same physical stimuli.

Finally, it is worth noting that there were consistent individual differences in the size of intermodal mismatch (recall the comparisons made between Experiments 2 and 3 ). They are indications of the reliability of measurements, and they are interesting in their own right as a probable route to better understanding of the mechanism of crossmodal matching.

\section{Orderly Ratios}

The apparently equal sizes are related by power functions. This is another aspect of data (the first aspect, discussed above, is the variability of intermodal mismatch): order within each condition and also on the individual level (power functions are equally valid descriptions on the individual level; see Appendix B). The exponents for different conditions varied from 0.961 (Experiment 4) to 1.23 (Experiment 2) for linear extents; for the circles, the exponent was 1.42 . Unlike in cross-modal matching functions relating stimuli intensities of different physical continua - that is, physical continua with different scales of measurement (e.g., line lengths and levels of a band of noise, as in Stevens \& Guirao, 1963)-constants are not arbitrary in these power functions, because they relate the same physical intensity mediated via two modalities. Together with the exponent, constants completely describe relationships between apparently equal visual and kinesthetic sizes - not only how their increments relate but also what the absolute differences between them are.

We may speculate that it is convenient to have regularity in the relationship between the visual and kinesthetic length percepts, in order to coordinate eye and hand in pursuing movements (as much as the perception of length by either modality plays a part in eye-hand coordination). In other words, we may consider the power relationship between vision and kinesthesia not only as a necessary outcome of power relationships governing percepts of length within modalities (information that is there) but as a feature of practical significance for our visuomotor coordination (information that we use).

How would we benefit if the latter is the case? We can make a simple analogy to the functional explanation of the power law governing perception of intensities. According to this explanation, in a changing environment, the ratios between our sensations remain constant for the constant ratios between stimuli intensities, allowing us to enjoy relative perceptual invariance (see, e.g., Yilmaz, 1967). In terms of eye-hand coordination, the variable "environment" would be variable conditions of movement (e.g., visual input, direction, orientation, and speed of movement) resulting in variable intermodal mismatch of lengths. The invariant feature would be the power relationship between them: The constant ratios of visual lengths correspond to the constant ratios of kinesthetic lengths, although the exact sizes of the corresponding ratios vary across conditions, and they probably have to be learned for each condition.

\section{Awareness of the Mismatch}

The final question we will consider is this: Given the possible discrepancies between the kinesthetic and visual length percepts in everyday situations, why are we not aware of them? For example, when we draw a line of about $30 \mathrm{~cm}$, we can see the distance moved, and we can feel it via kinesthesia. According to the results of Experiment 1, the intermodal discrepancy is there, but we do not normally experience it.

The important difference is that, in everyday situations, the perception is multimodal. The studies of adaptation of space perception show what happens when there is an intermodal conflict in multimodal perception (for a review, see Welch, 1986). The subjects are confronted with a discrepancy between or within the spatial modalities. Discrepancies are artificially induced and often large. For example, subjects wear prisms that displace the visual field to one side, and they are asked to perform simple pointing tasks. Several mechanisms are said to contribute to the resolution of these situations of conflict. Most often, the percept obtained by one of the modalities undergoes some modification.

It appears that the modality that reveals the primary modification when confronted with perceptual rearrangement is generally the one not being attended. Thus when vision and proprioception are placed in conflict, it is typically vision that is attended and thus proprioception that undergoes the major alteration. It seems likely that the modality to which the observer typically pays more attention is the one assumed (not necessarily consciously) to be the more veridical or trustworthy. That this modality is frequently vision may be due, at least in part, to its high degree of precision and general adeptness at spatial tasks. (Welch, 1986, p. 24-37)

Almost a century earlier, William James (1890) reached an almost identical conclusion: "When two sensorial space- 
impressions, believed to come from the same object, differ, then THE ONE MOST INTERESTING, practically or esthetically, IS JUDGED TO BE THE TRUE ONE" (p. 181; italics and capitals by James).

Artificially introduced discrepancies in adaptation experiments may be considered extreme relative to discrepancies that sometimes exist between normal, undistorted kinesthetic and visual size percepts. But, there are also studies of natural conflict situations. For example, in R. Teghtsoonian and M. Teghtsoonian's (1970) study, subjects simply held an object in the hand looking at it at the same time. The size estimates they made were more like previously obtained visual estimates than proprioceptive ones. The authors conclude that, in the natural conflict between visual and tactual information, the subjects relied on the visual input. However, we are reminded by James (1890) and Welch (1986) not to jump to the conclusion that vision always dominates.

\section{REFERENCES}

AVERY, G. C., \& DAY, R. H. (1969). Basis of horizontal-vertical illusion. Journal of Experimental Psychology, 81, 376-380.

Brown, J. S., Knauft, E. B., \& Rosenbaum, G. (1948). The accuracy of positioning reactions as a function of their direction and extent. American Journal of Psychology, 61, 167-182.

Cheng, M. F. H. (1968). Tactile- kinesthetic perception of length. American Journal of Psychology, 81, 74-82.

ClaRK, F. J., \& HoRCH, K. W. (1986). Kinaesthesia. In K. R. Boff, L Kaufman, \& J. P. Thomas (Eds.), Handbook of perception and human performance (pp. 13-1 to 13-62). New York: Wiley.

ConNOLly, K., \& JoNEs, B. (1970). A developmental study of afferentreafferent integration. British Journal of Psychology, 61, 259-266.

CoREN, S., \& GiRGus, J. S. (1978). Seeing is deceiving: The psychology of visual illusions. New York: Erlbaum.

Hatwell, Y. (1990). Spatial perception by eyes and hand: Comparison and intermodal integration. In C. Bard, M. Fleury, \& L. Hay (Eds.), Development of eye-hand coordination across the life span (pp. 99-132). Columbia: University of South Carolina Press.

JAMES, W. (1890). Principles of psychology. New York: Holt.

JASTROW, J. (1886). The perception of space by disparate modalities. Mind, 11, 539-554.

JONES, B. (1973). When are vision and kinaesthesis comparable? British Journal of Psychology, 64, 587-591.

KuENNEPAS, T. M. (1955). An analysis of the "horizontal-vertical illusion." Journal of Experimental Psychology, 49, 134-140.

Laszlo, J. I., BEGG, J. M., \& SaInsbury, K. M. (1994). The size illusion in children from five years of age and adults. Perception, 23, 201-206.

LASZLO, J. I., \& BrodERICK, P. (1985). The size illusion: Visual and kinaesthetic information in size perception. Perception, 14, 285-291.

LoOMIS, J. M., \& Lederman, S. J. (1986). Tactual perception. In K. R. Boff, L. Kaufman, \& J. P. Thomas (Eds.), Handbook of perception and human performance (pp. 31-1 to 31-4I). New York: Wiley.

MARKS, L. E. (1978). The unity of the senses: Interrelations among the modalities. New York: Academic Press.

Michaels, C., \& Carello, C. (1981). Direct perception. Englewood Cliffs, NJ: Prentice-Hall.

Millar, S. (1972). The development of visual and kinesthetic judgments of distance. British Journal of Psychology, 63, 271-282.

Newell, K. M., Shapiro, D. C., \& Carlton, M. J. (1979). Coordinating visual and kinesthetic memory codes. British Journal of Psychology, 70, 87-96.

ONO, A. (1969). Interdependence in successive judgements of the duration, distance and speed of a manual movement. Tohuku Psychologica Folia, 28, 29-53.

Paillard, J., \& Brouchon, M. A. (1974). A proprioceptive contribu- tion to the spatial encoding of position cues for ballistic movements. Brain Research, 71, 273-284.

RAFFEL, G. (1936). Visual and kinesthetic judgments of length. American Journal of Psychology, 48, 331-334.

Ronco, P. G. (1963). An experimental quantifications of kinesthetic sensation: Extent of arm movement. Journal of Psychology, 55, 227-238

RuLE, S. J. (1969). Subject difference in exponents from circle size, numerousness, and line length. Psychonomic Science, 15, 284-285.

SeIzova, T. (1995). Visual, tactual, kinesthetic and haptic size perception: Cross-modal matching (Report No. 17, pp. 1-28). Belgrade: University of Belgrade, Laboratory for Experimental Psychology.

Seizova, T., \& Stankov, L. (1998). Individual differences in crossmodal matching of length. Manuscript in preparation.

Stevens, S. S. (1957). On the psychophysical law. Psychological Review, 64, 153-181.

Stevens, S. S. (1971). Issues in psychophysical measurement. Psychological Review, 78, 426-450.

STEvens, S. S., \& Guirao, M. (1963). Subjective scaling of length and area and the matching of length to loudness and brightness. Journal of Experimental Psychology, 66, 177-186.

TEGHTSOONIAN, M. (1965). The judgment of size. American Journal of Psychology, 78, 392-402.

TEGHTSOONIAN, R. (1981). Logical difficulties in physical correlate theory. Behavioral \& Brain Sciences, 4, 205-206.

Teghtsoonian, R., \& Teghtsoonian, M. (1970). Two varieties of perceived length. Perception \& Psychophysics, 8, 389-392.

WELCH, R. B. (1986). Adaptation of space perception. In K. R. Boff, L. Kaufman, \& J. P. Thomas (Eds.), Handbook of perception and human performance (pp. 24-1 to 24-37). New York: Wiley.

WoNG, T. S. (1977). Dynamic properties of radial and tangentıal movements as determinants of the haptic horizontal-vertical illusion with an L figure. Journal of Experimental Psychology: Human Perception \& Performance, 3, 151-164.

YiLMAZ, H. (1967). Perceptual invariance and the psychophysical law. Perception \& Psychophysics, 2, 533-538.

ZELAZNIK, H. N., \& LANTERO, D. (1996). The role of vision in repetitive circle drawing. Acta Psychologica, 92, 105-118.

\section{NOTE}

1. Brown, Knauft, and Rosenbaum's (1948) study was not designed to address the question of length perception, but it is included because the results are of interest anyway (see the General Discussion section). On the other hand, there are cross-modal matching studies of length that are not cited in Table 1. Their main concern was the precision of intermodal matches measured by absolute error. The signed differences between matches are rarely reported, and not in detail. The motivation for most of these studies was testing the implications of the theories of intermodal development (for a review, see Hatwell, 1990).

\section{APPENDIX A \\ Modes of Presentation of Standards and Matches in the Studies on Perception of Linear Stimuli Perceived by Vision and by Movement}

\section{Visual Stimuli Presentation}

In Jastrow's (1886) study, lines drawn on a paper were shown through the frame; a series of lines was presented, each differing in length from its neighbor; and subjects marked an appropriate length on the long line drawn on a paper. In Raffel's (1936) study, subjects were shown a piece of cardboard. In Laszlo et al.'s (1994) study, lines were drawn in random positions on the board. In the studies of Jones (1973) and Millar (1972), a lit bulb was moved to the required distance. In Brown et al.'s (1948) study, the target, a marker, was placed at a certain distance from another marker. In Seizova's (1995) study, two dots were drawn on a paper, placed at a certain distance from each other. 


\section{Manner of Movement}

In most studies, a special apparatus was used to control the movement length. It usually consisted of tracks with a movable part, with a rod or stylus, or without it. Subjects held this movable part while performing a movement (Connolly \& Jones, 1970; Jastrow, 1886; Jones, 1973; Laszlo et al., 1994; Millar, 1972). In some studies, the movement was passive, subjects' hands being taken along the track (Jones, 1973; Laszlo et al., 1994). The other ways of moving were drawing (Jastrow, 1886; Seizova, 1995) and free arm movement through the air (Seizova, 1995). In one study (Raffel, 1936), kinesthesis was combined with touch: Subjects ran the finger along the edge of the cardboard to feel it's length.

\section{APPENDIX B}

\section{Individual Power Functions}

Individual best-fitting power functions were calculated on the individual means (based on three trials) from Experiment 2 in the frontoparallel condition (this condition was arbitrarily chosen). They relate apparently equal visual (V) and kinesthetic (K) lengths. Power functions and $r^{2}$ values for 16 subjects were as follows:
1. $\mathrm{V}=0.075 \mathrm{~K}^{1.465}, r^{2}=.999$

2. $\mathrm{V}=0.160 \mathrm{~K}^{1.375}, r^{2}=.979$

3. $\mathrm{V}=0.652 \mathrm{~K}^{1189}, r^{2}=.947$

4. $\mathrm{V}=0.255 \mathrm{~K}^{1.228}, r^{2}=.992$

5. $\mathrm{V}=0.088 \mathrm{~K}^{1.486}, r^{2}=.997$

6. $\mathrm{V}=0.336 \mathrm{~K}^{1.256}, r^{2}=.997$

7. $\mathrm{V}=0.073 \mathrm{~K}^{1.479}, r^{2}=.995$

8. $\mathrm{V}=0.183 \mathrm{~K}^{1.291}, r^{2}=.978$

9. $\mathrm{V}=0.527 \mathrm{~K}^{1107}, r^{2}=.995$

10. $\mathrm{V}=0.847 \mathrm{~K}^{1.048}, r^{2}=.995$

11. $\mathrm{V}=0.557 \mathrm{~K}^{1131}, r^{2}=.979$

12. $\mathrm{V}=0.123 \mathrm{~K}^{1.379}, r^{2}=.983$

13. $\mathrm{V}=0.988 \mathrm{~K}^{0.981}, r^{2}=.998$

14. $\mathrm{V}=0.393 \mathrm{~K}^{1.211}, r^{2}=.965$

15. $\mathrm{V}=0.517 \mathrm{~K}^{1160}, r^{2}=.988$

16. $\mathrm{V}=0.555 \mathrm{~K}^{1170}, r^{2}=.996$.

(Manuscript received May 30, 1996; revision accepted for publication June 10, 1997.) 